\title{
Competitive Online Algorithm for Leasing Wireless Channels in 3-Tier Sharing Framework
}

\author{
Gourav Saha, Alhussein A. Abouzeid, and Marja Matinmikko
}

\begin{abstract}
To meet the ever growing need for wireless spectrum, the Federal Communication Commision (FCC) introduced a spectrum sharing model called the 3-Tier Sharing Framework. In this model, under-utilized federal spectrum will be released for shared use where the highest preference will be given to Tier-1 followed by Tier-2 and then Tier-3. In this paper, we present a model where a wireless operator, who is interested in maximizing its profit, can operate as a Tier-2 and/or a Tier-3 user. Tier-2 is characterized by paid but "almost" guaranteed and interference free channel access while Tier-3 access is free but has lesser guarantee and also faces channel interference. So the operator has to optimally decide between paid but better channel quality and free but degraded channel quality. Also, the operator has to make these decisions without knowing future market parameters like customer demands or channel availability. We use tools from ski-rental literature to design a deterministic online algorithm for leasing channels which does not rely on the knowledge of market statistics. The efficiency of the online algorithm is analyzed by deriving its competitive ratio (CR) and by conducting simulations. The mathematical model for leasing channels is a novel generalization of the classical skirental problem. We therefore make fundamental contribution to ski-rental literature which may have diverse applications beyond the problem considered in this paper.
\end{abstract}

\section{INTRODUCTION}

The demand for wireless Internet access is ever growing and the wireless spectrum is getting scarce. The President's Council of Advisors on Science and Technology (PCAST), in their report [1], calls the notion of spectrum scarcity a "fundamental misunderstanding" arising due to underutilization of spectrums. In support of PCAST report [1], the FCC decided to release $150 \mathrm{MHz}$ of federal spectrum (from 3.55 to $3.7 \mathrm{GHz}$ ). The shared use of these federal spectrums should follow the 3-Tier Sharing Framework [2] (refer Figure 1): Tier-1 is called the "Incumbent tier" consisting of federal users who have the highest priority access to any channel and are guaranteed interference protection from lower tiers. Tier-2 is called the "Priority Access Licenses (PAL) tier". PAL users can lease the channels by participating in auctions. They can use the leased channels whenever Tier-1 users are not using it. Priority Access Users are gauranteed interference protection from Tier-3 users. Tier-3 is called the "Generalized Authorized Access (GAA) tier". GAA users can opportunistically use a channel for free provided that it is not used by Tier-1 or Tier- 2 users. A Tier-3 user is

This work was supported in part by National Science Foundation grant CNS-1456887 and Tekes in JoiNtMaCS project. G. Saha and A. A. Abouzeid are with the Department of Electrical, Computer and Systems Engineering, Rensselaer Polytechnic Institute, Troy, NY 12180, USA; Email: sahag@rpi.edu, abouzeid@ecse.rpi.edu. M. Matinmikko is with Centre for Wireless Communications (CWC), University of Oulu, Finland; Email: Marja.Matinmikko@ee.oulu.fi.

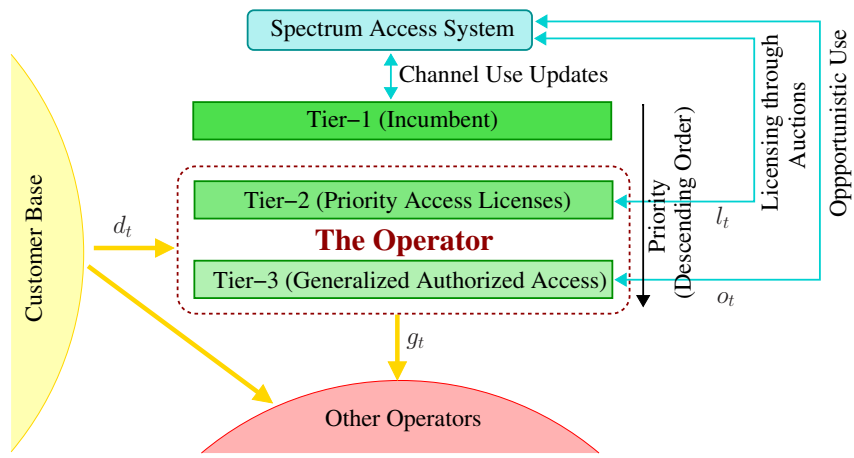

Fig. 1. The 3-Tier Sharing Framework and our System Model.

not gauranteed interference protection from Tier-1, Tier-2 or even other Tier-3 users. The Spectrum Access System (SAS) is a central database which keep record of channel states. It is also a policy engine which enforces the 3-Tier hierarchy.

In this paper, we consider a market consisting of many operators. These operators use wireless spectrum to serve customer demands. We consider one such operator, labelled "The Operator" in Figure 1. The objective is to maximize the profit of the operator. In our model, the operator can work as Tier-2 and/or Tier-3 user. We consider a time slotted model where at the $t^{t h}$ epoch, the operator must make the following three decisions upon receiving $d_{t}$ customer demands:

1) Amount of customer demand $g_{t}$ to reject. As shown in Figure 1, the rejected customer demands gets served by "other operators" in the market.

2) Number of channels $l_{t}$ to lease (Tier-2) in order to serve the accepted customer demands.

3) Number of channels $o_{t}$ to use opportunistically (Tier-3) in order to serve the accepted customer demands. 2

The decision process of rejecting demands, leasing channels and opportunistic channel use relies on customer demand pattern and channel availability trends. The operator has to make decisions without the future knowledge of these variables. Online nature of the problem leads to the following uncertainties when an operator wants to lease a channel:

1) Uncertainty in Customer Demand: Leasing a channel is profitable only if the customer demand in future epochs is consistently high.

2) Uncertainty in channel availability for opportunistic use: Leasing a channel is not profitable if there are enough channels for opportunistic use in future epochs.

3) Uncertainty in channel availability for leasing: All the channel leases may get sold out in future.

Along with the online nature of the problem, there is 
an additional issue that serving customer demands using opportunistic channels may lead to lower Quality of Service (QoS) to the customers because such channels may suffer from harmful interference. This is a challenging online optimization problem which we address in this paper.

To the best of authors knowledge, the only work in spectrum sharing literature which resembles our problem is addressed in [3]. In [3], the authors modelled the customer demand and channel availability statistics as Discrete Time Markov Chain. It then used tools from Stochastic Dynamic Programming to design an online algorithm for leasing channels. Apart from the problem considered in this paper, there are other bodies of work which is of importance to the 3-TSF. In [4], the authors designed a network protocol and a SAS which implements the rules of the 3-TSF. The work done in [5] considers a market where an operator can operate in either Tier- 2 or Tier-3. It investigates the incentive of an operator to enter such a market in presence of competition. Other allied areas of research can be of significant importance to the 3-TSF though they are not directly related. From economic standpoint, research in the field of spectrum contracts ([6], [7]), auctions and pricing ([8]) will help understand if the 3-Tier Sharing Framework is economically attractive for potential investors. From technical standpoint, dynamic channel allocation is of significant importance to 3-TSF. While doing dynamic channel allocation it is crucial to consider blocking probability [9] and co/adjacent channel interference [10].

This paper makes three contributions. First, we present a system model in which an operator using 3-TSF can maximize its profit by strategically operating as a Tier-2 and/or a Tier-3 user. Our model is novel as it captures key elements of 3-TSF.

Second, compared to [3], our online algorithm has two novelty. First, unlike [3], our algorithm does not require statistical knowledge of the involved random processes (customer demand and spectrum availability). In the early stages of the deployment of 3-TSF, the operator will have either limited or no knowledge of market statistics. Hence, the algorithm proposed in this paper will be more suitable compared to [3]. Second, the algorithm proposed in [3] has high time complexity (pseudo-polynomial) if the optimization horizon is large. Our algorithm has low time complexity (polynomial) irrespective of the optimization horizon.

Third, this work adds a new application area to the skirental problem among the already existing ones [11], [12], [13]. We also make a fundamental contribution to ski-rental literature, i.e. how the classical breakeven algorithm for the ski-rental problem gets modified if the number of skis available for leasing is finite.

The rest of the paper is organized as follows: In section II we mathematically formulate the profit maximization problem of the operator. Section III contains the main result of the paper, a competitive deterministic online algorithm for leasing channels. Simulation results are presented in Section IV. Finally we conclude the paper in Section V with a brief discussion on the immediate extensions to this work.
TABLE I

A TABLE OF FREQUENTLY USED NOTATIONS.

\begin{tabular}{|c|c|}
\hline Notation & Description \\
\hline$T$ & Optimization Horizon. \\
\hline$M$ & Total number of channels. \\
\hline$d_{t}$ & $\begin{array}{l}\text { Number of channels needed to serve the customer demand at } \\
\text { epoch } t \text {. We call this "demand" in the rest of the paper. }\end{array}$ \\
\hline$g_{t}$ & Number of demands the operator rejected at epoch $t$. \\
\hline$p_{t}$ & Price of serving a demand at epoch $t$ \\
\hline$p_{M}$ & Upper bound on $p_{t}, p_{t} \leq p_{M}$ \\
\hline$P, \tau$ & Price and duration of a channel lease. In general, $p_{t} \ll P$. \\
\hline$M_{t}^{l}$ & Number of channels available for leasing at epoch $t$. \\
\hline$l_{t}$ & Number of channels the operator leased at epoch $t$. \\
\hline$v_{t}$ & Number of channels other operators wants to lease at epoch $t$. \\
\hline$w_{t}$ & Number of channels leased by all the operators at epoch $t$. \\
\hline$M_{t}^{o}$ & $\begin{array}{l}\text { Number of channels the operator can opportunistically use at } \\
\text { epoch } t \text {. }\end{array}$ \\
\hline$A_{t}$ & Number of active channel leases the operator has at epoch $t$. \\
\hline$\lambda_{t}$ & $\begin{array}{l}\text { Number of active channel leases which got pre-empted by Tier- } \\
1 \text { users at epoch } t \text {. }\end{array}$ \\
\hline$o t$ & $\begin{array}{l}\text { Number of channels the operator used opportunistically at } \\
\text { epoch } t \text {. }\end{array}$ \\
\hline$f_{t}(o t)$ & A function to penalize opportunistic channel use. \\
\hline$r_{t}$ & $\begin{array}{l}r_{t}=g_{t}+o_{t} \text {, the combined action of using channels } \\
\text { opportunistically and rejecting demands. }\end{array}$ \\
\hline$F_{t}\left(r_{t}\right)$ & Renting function: A function to penalize the renting action. \\
\hline$\varphi_{t}$ & $\begin{array}{l}\varphi_{t}=\left(d_{t}, \lambda_{t}, p_{t}, M_{t}^{o}, v_{t}, f_{t}(\cdot)\right) \text {, a tuple which forms the } \\
\text { input to OP1. }\end{array}$ \\
\hline$(x)^{+}$ & Positivity operator: $(x)^{+}=\max (0, x)$ \\
\hline
\end{tabular}

\section{System ModeL}

Notations: Table I lists notations used throughout this paper. Other notations used in this paper are standard.

In this section we formulate the profit maximization problem of the operator. We also discuss the underlying assumptions in our problem formulation. Time is considered to be slotted. In every epoch $t \in\{1, \ldots, T\}$, the decision variables are $g_{t}, o_{t}, l_{t} \in\{0, \ldots, M\}$. Operator's profit is

$\mathcal{P}=\sum_{t=1}^{T}\left(p_{t}\left(d_{t}-g_{t}\right)-P l_{t}\right)=\underbrace{\sum_{t=1}^{T} p_{t} d_{t}}_{1^{\text {st }} \text { term }}-\underbrace{\sum_{t=1}^{T}\left(p_{t} g_{t}+P l_{t}\right)}_{2^{\text {nd }} \text { term }}$

where $p_{t}\left(d_{t}-g_{t}\right)$ is the operator's revenue for serving $\left(d_{t}-g_{t}\right)$ demands and $P l_{t}$ is the operator's expense to buy $l_{t}$ channels at epoch $t$. Operator wants to maximize its profit $\mathcal{P}$ which is equivalent to minimizing the $2^{\text {nd }}$ term because in our model, the operator has no control demand $d_{t}$ and price $p_{t}$. To make the model more realistic, we add a function $f_{t}\left(o_{t}\right)$ to the $2^{n d}$ term which penalizes opportunistic use of channels. This leads to the final optimization problem

$$
\begin{array}{cl}
\text { OP1 : } & \min _{\left\{g_{t}, o_{t}, l_{t}\right\}} \mathcal{C}=\sum_{t=1}^{T}\left(p_{t} g_{t}+f_{t}\left(o_{t}\right)+P l_{t}\right) \\
\text { subject to: } & o_{t}+g_{t}+\left(\sum_{i=(t-\tau+1)}^{t} l_{i}-\lambda_{t}\right) \geq d_{t} \\
& 0 \leq o_{t} \leq M_{t}^{o} ; 0 \leq l_{t} \leq M_{t}^{l} ; 0 \leq g_{t}
\end{array}
$$

The function $f_{t}\left(o_{t}\right)$ is time-varying and is assumed to be convex and increasing in the range $\left[0, M_{t}^{o}\right]$. It has two real 
world interpretations: First, to account for harmful interference in an opportunistic channel, the operator may choose to transmit at a higher power level ${ }^{1}$. In this case $f_{t}\left(o_{t}\right)$ respresents the cost to transmit at a higher power level. Second, the use of opportunistic channels leads to lower QoS to the customers if the channel interference is high. The function $f_{t}\left(o_{t}\right)$ can be used to capture the monetary loss due to lower QoS. The term $\sum_{i=(t-\tau+1)}^{t} l_{i}$ in OP1 is the number of active channel leases at epoch $t$ and is denoted as $A_{t}$. However $\lambda_{t}$ active leases may get interrupted by Tier-1 users ${ }^{2}$ leaving effectively $\left(\sum_{i=(t-\tau+1)}^{t} l_{i}-\lambda_{t}\right)$ active channel leases. Given that the lease period of a channel is $\tau$, the time evolution of $M_{t}^{l}$ is governed by the equation ${ }^{3}$

$$
\begin{array}{rlr}
M_{t}^{l} & =M_{t-1}^{l}-w_{t-1}+w_{t-\tau} \quad \text { where } \\
w_{t} & =l_{t}+\min \left(M_{t}^{l}-l_{t}, v_{t}\right)
\end{array}
$$

where $w_{t-\tau}$ are the number of leases which reappears in the market at $t^{t h}$ epoch when its lease period expires. In equation (3), the term $\min \left(M_{t}^{l}-l_{t}, v_{t}\right)$ is the number of channels leased by other operators in the market. This term captures an key assumption of our model, i.e. the operator has superior auctioning strategy compared to other operators. Therefore if the operator wants to lease $l_{t} \leq M_{t}^{l}$ channels at epoch $t$, it will win the bid for $l_{t}$ channels, leaving $M_{t}^{l}-l_{t}$ channels which can be leased by the other operators.

In our model, the tuple $\varphi_{t}$ (defined in Table I) is a time sequence which forms the input to $\mathbf{O P} 1$. The cost $\mathcal{C}$ incurred by $\mathbf{O P} 1$ is a function of $\varphi_{t}$. All other variables in OP1, except $p_{t}$ and $P$, lies in the discrete set $\{0,1, \ldots, M\}$.

\section{A. Assumptions}

The key assumptions in our system model are as follows:

1) The cost of leasing a channel is time varying as it depends on the outcome of the auction. However we assumed it to be a constant $P$.

2) The role of competition among operators to maximize the profit of the operator is not considered., for e.g. we do not design optimal pricing policy for $p_{t}$ to compete for customer demand nor do we consider optimal bidding strategy to compete in auctions. In our model $p_{t}$ and $d_{t}$ are arbitrary time varying sequences which can not be controlled by the operator.

Other than the assumptions on system model, we also need to impose the following assumptions in order to design online algorithms with provable theoretic bounds:

1) Future knowledge of $p_{t}, d_{t}, \lambda_{t}, M_{t}^{o}, v_{t}$ and $f_{t}(\cdot)$ is not assumed. However we impose the following constrain

$$
0<\mu_{l} \leq \frac{\sum_{i=(t-\tau+1)}^{t} M_{i}^{l}}{\tau} ; \forall t
$$

\footnotetext{
${ }^{1}$ The highest power level at which the operator can transmit as Tier-3 user is constrained by FCC rules. This indeed leads to the second interpretation.

${ }^{2}$ The SAS will try to relocate the channel of Tier-2 user if it gets interrupted by Tier-1 user. $\lambda_{t}$ models such relocations of channels too.

${ }^{3}$ Equation 2 and 3 is valid even for $t<1$. However $l_{t}=0 ; \forall t<1$.
}

which says that the moving horizon time average of $M_{t}^{l}$ over $\tau$ period is at least $\mu_{l}$.

2) The function $f_{t}\left(o_{t}\right)$ can be evaluated for any $o_{t}$.

3) $p_{t}$ is upper bounded by $p_{M}$, i.e. $p_{t} \leq p_{M} ; \forall t$. Knowledge of $p_{M}$ is assumed.

\section{Deterministic Online Algorithm}

In this section we will design a deterministic online algorithm for leasing channels. We approach this in steps. In Section III-A we introduce a theorem which provides better insight into the structure of OP1 and effectively reduces the number of decision variables from three to two. In Section III-B we consider a special case of OP1 called the Modified Ski-Rental Problem. It is simpler to analyze but provides useful insights into the online algorithms for OP1. Finally, we will design and analyze a deterministic online algorithm for leasing channels in Section III-C.

\section{A. Simplification of $\mathbf{O P 1}$}

OP1 can be decoupled into two sub-problems, one which decides how many of the available opportunistic channel to use and the other which captures the online nature of leasing channels. The following theorem formalizes this notion.

\section{Theorem 1: Let}

$$
\text { OP2 : } \bar{o}_{t}=\underset{0 \leq o_{t} \leq M_{t}^{o}}{\arg \min }-p_{t} o_{t}+f_{t}\left(o_{t}\right)
$$

and define the following function

$$
F_{t}\left(r_{t}\right)=p_{t}\left(r_{t}-\bar{o}_{t}\right)^{+}+f_{t}\left(\min \left(r_{t}, \bar{o}_{t}\right)\right)
$$

Then the optimal solution $g_{t}^{*}, o_{t}^{*}$ and $l_{t}^{*}$ of $\mathbf{O P} 1$ can be obtained by solving the optimization problem

$$
\begin{array}{cl}
\text { OP3 : } & \min _{\left\{r_{t}, l_{t}\right\}} \mathcal{C}=\sum_{t=1}^{T}\left[F_{t}\left(r_{t}\right)+P l_{t}\right] \\
\text { subject to: } & r_{t}+\sum_{i=(t-\tau+1)}^{t} l_{i} \geq D_{t} \triangleq\left(d_{t}+\lambda_{t}\right) \\
& 0 \leq l_{t} \leq M_{t}^{l} ; 0 \leq r_{t}
\end{array}
$$

for the optimal solution $\bar{r}_{t}$ and $\bar{l}_{t}$ and then setting

$$
g_{t}^{*}=\left(\bar{r}_{t}-\bar{o}_{t}\right)^{+} ; \quad o_{t}^{*}=\min \left(\bar{r}_{t}, \bar{o}_{t}\right) ; \quad l_{t}^{*}=\bar{l}_{t}
$$

Proof: Please refer [14] for the proof.

OP2 decides how many of the available opportunistic channel $M_{t}^{o}$ to be used at epoch $t$. The function $h_{t}\left(o_{t}\right)=$ $-p_{t} o_{t}+f_{t}\left(o_{t}\right)$ is unimodal (refer [14]). We can therefore use tools like binary search or fibonnaci search [15] to solve OP2 in $\mathcal{O}\left(\log _{2}\left(M_{t}^{o}\right)\right)$ time.

OP3 has two decision variables. The variable $l_{t}$ as usual implies leasing (Tier-2). The new variable $r_{t}$ implies renting. Renting is the combined action of using channels opportunistically (Tier-3) and rejecting demands, i.e. $r_{t}=o_{t}+g_{t}$. So in every epoch the operator has to decide how much to rent and how much to lease in order to serve the effective demand $D_{t}$. We will use this terminology quite often in rest of the paper. The function $F_{t}\left(r_{t}\right)$ is called the renting function. It penalizes the renting action and has the following properties: 
Property 1: $F_{t}\left(r_{t}\right)$ is monotonically increasing in $r_{t}$. This property suggest that if $\bar{l}_{t}$ is the optimal solution to OP3, and $\bar{A}_{t}$ the corresponding sequence of the number of active leases, then the optimal solution $\bar{r}_{t}$ is given by

$$
\bar{r}_{t}=\left(D_{t}-\bar{A}_{t}\right)^{+}
$$

Property 2: $F_{t}\left(r_{t}\right)$ is convex in $r_{t}$. This implies

$$
F_{t}\left(r_{t}\right)-F_{t}\left(r_{t}-1\right) \leq F_{t}\left(r_{t}+1\right)-F_{t}\left(r_{t}\right) ; \forall r_{t}
$$

Property 3: First derivative of $F_{t}\left(r_{t}\right)$ is bounded as follows

$$
F_{t}\left(r_{t}+1\right)-F_{t}\left(r_{t}\right) \leq p_{t} \leq p_{M} ; \forall r_{t}
$$

The proof of these properties are trivial. It can be found in [14] but has been skipped here for brevity.

OP3 captures the online nature of leasing channels. Say that the operator has to decide the optimal number of lease $\bar{l}_{t}$ at epoch $t$. A necessary condition for optimality of $\bar{l}_{t}$ is

$$
\sum_{i=t}^{t+\tau-1}\left[F_{i}\left(\left(D_{i}-a_{i}\right)^{+}\right)-F_{i}\left(\left(D_{i}-a_{i}-\bar{l}_{t}\right)^{+}\right)\right] \geq P \bar{l}_{t}
$$

where $a_{i}=\sum_{j=i-\tau+1}^{t-1} \bar{l}_{j} ; \forall i \in\{t, \ldots, t+\tau-1\}$ is the number of active leases in the $i^{t h}$ epoch if $\bar{l}_{t}=0$. Inequality (10) implies that the net rental cost saved by leasing $\bar{l}_{t}$ channels should be greater than the cost of leasing $\bar{l}_{t}$ channels. The operator must know $D_{i}, F_{i}(\cdot) ; \forall i \in\{t, \ldots, t+\tau-1\}$ in order to choose a $\bar{l}_{t}$ which satisfies inequality (10). To calculate $D_{i}, F_{i}(\cdot)$ for $i>t$, the operator needs future knowledge of $\varphi_{i}$ (refer Table I). Hence, online information is not enough to find an optimal sequence $\bar{l}_{t}$.

The operator has to decide $\left(r_{t}, l_{t}\right)$ just based on the knowledge of $\varphi_{i} ; \forall i \leq t$ in a certain optimal sense called the competitive ratio. Competitive ratio $(\mathrm{CR})$ is a relative measure of an online algorithm with respect to an optimal algorithm ${ }^{4}$. Define the sequence $\varphi=\left\{\varphi_{1}, \varphi_{2} \ldots, \varphi_{T}\right\}$. Let $\mathcal{C}_{\mathcal{A}}(\varphi)$ and $\mathcal{C}_{O P T}(\varphi)$ be the cost incurred by a deterministic online algorithm $\mathcal{A}$ and the optimal algorithm $O P T$ respectively. $\mathcal{A}$ is called $c$-competitive iff

$$
\mathcal{C}_{\mathcal{A}}(\varphi) \leq c \cdot \mathcal{C}_{O P T}(\varphi) ; \forall \varphi \in \mathcal{S}
$$

A smaller $c$ implies a better online algorithm. The set $\mathcal{S}$ contains all possible values of $\varphi$. Competitive analysis is often thought of as a two player game between an adversary which generates $\varphi$ to maximize the ratio $\frac{\mathcal{C}_{\mathcal{A}}(\varphi)}{\mathcal{C}_{O P T}(\varphi)}$ and the online algorithm $\mathcal{A}$ which tries to minimize the ratio.

The rest of this section deals with designing an online algorithm for OP3. OP3 has important resemblance with works in ski-rental literature like [12] but with one key difference: the operator cannot lease more than $M_{t}^{l}$ channels at epoch $t$. This additional constrain makes the online algorithm for OP3 non-trivial both in terms of design and analysis.

\footnotetext{
${ }^{4}$ The optimal algorithm for OP3 is an offline algorithm based on dynamic programming and has pseudo-polynomial time complexity [12].
}

\section{B. Modified Ski-Rental Problem}

In this section we consider a modification of the classical Ski-Rental Problem (SRP) and show that it is a special case of OP3. Then we will design an optimal deterministic online algorithm to solve the Modified Ski-Rental Problem (MSRP) which will give us insights into solving OP3 online.

MSRP can be stated as follows:

1. A skier plans a skiing vacation with a tourism agency which rents a ski ${ }^{5}$ for $p_{M}$ dollars per day and leases a ski for $P$ dollars (where $p_{M} \ll P$ ) with the lease period being $\tau>1$ days $^{6}$. In context of OP3 it means that $F_{t}\left(r_{t}\right)=p_{M} r_{t}$.

2. The skier needs one ski a day. Skiing vacation is at most $\tau$ days (equal to the lease period) but can end on the $y^{\text {th }}$ day (where $0 \leq y \leq \tau$ ) if the skier gets injured while skiing. In context of OP3 it implies the following demand structure : $D_{t}=1 ; 1 \leq t \leq y$ and $D_{t}=0 ; t>y$.

3. The tourism agency has a maximum of $M$ skis to lease. The number of skis available for leasing on the $t^{t h}$ day is $M_{t}^{l}$ where $M_{t}^{l}$ is governed by equation 2 and 3. In context of MSRP, $l_{t}$ and $v_{t}$ are the number of skis "the skier" and the other skiers wanted to lease in day $t$ respectively.

4. The skier can lease a ski on the first day, i.e. $M_{1}^{l}>0$.

The above four points shows that MSRP is a special case of OP3. If $M=\infty$ then $M_{t}^{l}>0 ; \forall t$. In this case MSRP reduces to SRP. For SRP, the optimal online deterministic algorithm is the breakeven algorithm which states: Say the skier is still skiing on the $k^{\text {th }}$ day. If the net renting cost $p_{M} k \geq P$, the skier should lease a ski on the $k^{t h}$ day. Else, the skier should rent. CR of this algorithm is 2 .

If $M$ is finite then it is possible that $M_{t}^{l}=0$ for some $t$. The key difference between SRP and MSRP is the availability of ski leases. The skier may decide to lease on the $k^{t h}$ day only to find that $M_{k}^{l}=0$. Without any constrain on $M_{t}^{l}$, there may not be any skis available for leasing till the end of skier's vacation. In the worst case scenario, the skier has to keep renting till her vacation ends incurring a cost of $\tau p_{M}$ while the offline algorithm which can foresee the future will lease a ski on the $1^{\text {st }}$ day. Hence the CR is $\frac{\tau p_{M}}{P}$. This suggests that CR for MSRP cannot be better than $\frac{\tau p_{M}}{P}$ without any constrain on $M_{t}^{l}$. We therefore constrain $M_{t}^{l}$ using inequality (4). This leads to the following proposition.

Proposition 1: Say that the skier/operator decides to buy $l_{t}>0$ leases at epoch $t$. The skier/operator buys $l_{t}$ leases as and how it reappears in the market. Define

$$
\eta=\inf \left\{\delta \geq 0 \mid \sum_{k=0}^{\delta} M_{t+k}^{l} \geq l_{t}\right\}
$$

as the wait time because the skier/operator has to wait at least till epoch $t+\eta$ to buy all the $l_{t}$ leases. If $M_{t}^{l}$ is constrained by inequality (4), then $\eta$ can be upper bounded by $\eta_{M}$ where

$$
\eta_{M}=\tau\left(1-\frac{\mu_{l}}{M}\right) \leq \tau-1
$$

Proof: Please refer [14] for the proof.

\footnotetext{
5 "a ski" implicitly means a pair of skis.

6 "Renting" and "leasing" are indeed synonyms but in this paper they are differentiated based on price and contract duration.
} 
Proposition 2: Consider the following online algorithm:

1) Keep renting if the net renting cost is less than $z_{o p}$, where $z_{o p}$ is the solution to the quadratic equation

$$
z_{o p}^{2}+\tau\left(1-\frac{\mu_{l}}{M}\right) p_{M} z_{o p}-P^{2}=0
$$

If the net renting cost exceeds $z_{o p}$, the skier must decide to lease a ski.

2) If a ski is availabe for lease that day, then lease it. Else wait till it is available again.

3) The skier should buy a lease when it is available again only if the wait time $\eta \leq \tau-\frac{\left(z_{o p}+P\right)}{p_{M}}$. Else keep renting till the end of skiing vacation.

Among all online algorithms for MSRP which only assumes the knowledge of $\mu_{l}$ and hence the upper bound on wait time, $\eta_{M}=\tau\left(1-\frac{\mu_{l}}{M}\right)$, the above algorithm has the best CR of

$$
c_{o p t}\left(\mu_{l}\right)= \begin{cases}\left(1+\frac{z_{o p}}{P}\right)+\frac{\tau p_{M}}{P}\left(1-\frac{\mu_{l}}{M}\right) & ; \mu_{l} \geq \frac{M}{\left(\frac{\tau p_{M}}{P}-1\right)} \\ \frac{\tau p_{M}}{P} & ; \mu_{l}<\frac{M}{\left(\frac{\tau p_{M}}{P}-1\right)}\end{cases}
$$

Proof: Please refer [14] for the proof.

Theorem 2: An online algorithm for OP3 which only assumes the knowledge of $\mu_{l}$ and hence the upper bound on wait time, $\eta_{M}=\tau\left(1-\frac{\mu_{l}}{M}\right)$, cannot achieve CR better than $c_{\text {opt }}\left(\mu_{l}\right)$.

Proof: This follows from the fact that MSRP is a special case of OP3 and $c_{o p t}\left(\mu_{l}\right)$ is the best possible CR we can achieve for MSRP (by Proposition 2).

\section{Online Algorithm for Leasing Channels}

Motivated by the optimal online algorithm to solve MSRP, we suggest a threshold based algorithm for leasing channels. There are two threshold criterias:

1) The algorithm decides to lease a channel when the net incremental renting cost exceeds threshold $z_{t h}$.

2) The algorithm rejects the decision to lease a channel if the wait time exceeds the threshold $\tau-\frac{\left(z_{t h}+P\right)}{p_{M}}$.

A generic algorithm for any threshold $z_{t h}$ is presented in the listing Algorithm 1. However in this paper, we only concentrate on the case when $z_{t h}=P$. The working of the algorithm can be divided into five steps.

\section{Step 1 (Learn $\varphi_{t}$ )}

Recall that the tuple $\varphi_{t}=\left(d_{t}, \lambda_{t}, p_{t}, M_{t}^{o}, v_{t}, f_{t}(\cdot)\right)$ is the input to OP1. At epoch $t$, the operator knows $d_{t}$ and $p_{t}$ while $\lambda_{t}, M_{t}^{o}$ and $v_{t-1}$ can be learned by querying the $\mathrm{SAS}^{7}$. The penalty function $f_{t}(\cdot)$ for opportunistic channel use is estimated, possibly using QoS reviews from the customers.

\section{Step 2 (Calculate $\bar{o}_{t}$ )}

The operator computes $\bar{o}_{t}$ by solving OP2 using binary/ fibonacci search (line 6). The renting function $F_{t}\left(r_{t}\right)$ is

\footnotetext{
${ }^{7}$ Note that at time $t$, it is impractical to assume knowledge of $v_{t}$ however knowledge of $v_{t-1}$ is feasible. In many auction the demand of other bidders may be kept confidential because any asymmetry in information can lead to unfair advantage. However releasing such information after the auction may be feasible. We therefore assume that during time $t$, the operator can query SAS for the value of $l_{t-1}+v_{t-1}$ (the number of channels the entire market wanted to lease at time $t$ ) and hence infer $v_{t-1}$.
}

Algorithm $1 \mathcal{A}_{z_{t h}}$ : a deterministic online algorithm for leasing channels in 3-Tier Sharing Framework.

1. Let $x_{t}$ be the number of virtual active leases at the $t^{t h}$ epoch. Set $x_{t}=0 ; t=1,2, \ldots, T$

2. Let $y_{t}$ be the virtual number of channels available for leasing at the $t^{t h}$ epoch. Set $y_{t}=0 ; t=1,2, \ldots, T$

3. Initialize an empty FIFO queue which will store timestamps of leasing decisions.

4. Repeat steps 5-13 in all epochs. Let current epoch be $t$.

5. Learn $d_{t}, p_{t}, \lambda_{t}, M_{t}^{o}, v_{t-1}$ and $f_{t}(\cdot)$. Set $D_{t}=d_{t}+\lambda_{t}$.

6. Compute $\bar{o}_{t}$ by solving optimization problem OP2.

7. Set $w_{t-1}=\min \left(v_{t-1}, y_{t-1}\right), y_{t}=y_{t-1}-w_{t-1}+$ $w_{t-\tau}$.

8. Set $\mathcal{R}=z_{t h}$.

9. while $\left(\mathcal{R} \geq z_{t h}\right)$

9.a. Check the first epoch after $(t-\tau+1)$ when the operator could have bought another lease. Mathematically, $t_{M}=\inf \left\{k \mid y_{k}>0, k \geq t-\tau+1\right\}$.

9.b. Compute the net incremental rental cost $\mathcal{R}$ from $t_{M}$ to the current epoch $t$ using equation (13).

9.c. if $\left(\mathcal{R} \geq z_{t h}\right)$

9.c.1. The operator decides to lease a channel. Hence, current epoch $t$ is pushed into the FIFO queue.

9.c.2. Set $x_{i}=x_{i}+1 ; i=t_{M}, \ldots, t-1$ to update the history of $x_{i}$ 's. This shows that previous mistakes have been accounted.

9.c.3. Set $w_{t_{M}}=\min \left(w_{t_{M}}+1, y_{t_{M}}\right)$ and $y_{i}=\left(y_{i}-1\right)^{+} ; i=t_{M}, \ldots, t$. This shows that a virtual lease was bought at epoch $t_{M}$.

9.c.4. Set $x_{i}=x_{i}+1 ; i=t, \ldots, t+\tau-1$. This updates future $x_{i}$ 's to show that an additional virtual lease is available in future epochs.

\section{9.c. end if}

9. end while

10. Set the number of channels to lease to zero : $l_{t}=0$.

11. while $\left(M_{t}^{l}>0\right.$ AND "FIFO Queue is Not Empty")

11.a. Read timestamp from the FIFO queue. Let this epoch be $t_{l}$. Set wait time $\eta=t-t_{l}$.

11.b. if $\left(\eta \leq\left(\tau-\frac{\left(z_{t h}+P\right)}{p_{M}}\right)\right)$

11.b.1. Lease a channel: $l_{t}=l_{t}+1$.

11.b. end if

11.c. Pop timestamp from the FIFO queue.

11. end while

12. Number of active lease is $A_{t}=\sum_{i=t-\tau+1}^{t} l_{i}$. Remaining $r_{t}=\left(D_{t}-A_{t}\right)^{+}$demands are served by renting.

13. Number of channels to use opportunistically, $o_{t}=\min \left(r_{t}, \bar{o}_{t}\right)$. Reject $g_{t}=\left(r_{t}-\bar{o}_{t}\right)^{+}$demands.

implicitly dependent on $\bar{o}_{t}$ (refer equation (5)). Hence we need to compute $\bar{o}_{t}$ in order to evaluate $F_{t}\left(r_{t}\right)$ in Step 3.

Step 3 (Deciding to lease or not)

The operator maintains two time sequences $x_{t}$, the number of virtual active lease the operator has at epoch $t$ and $y_{t}$, the virtual number of channels available for leasing at epoch $t$. 


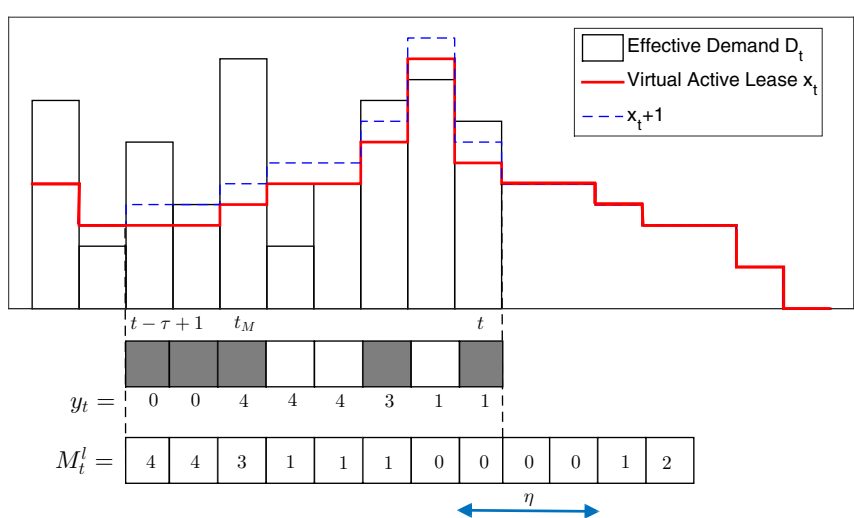

Fig. 2. An illustration of net incremental renting cost and wait time. When $x_{t}<D_{t}\left(x_{t} \geq D_{t}\right)$, i.e. the red graph is below (above) the black graph, a non-zero (zero) incremental renting cost is incurred. This is depicted using grey (white) epochs in the upper strip. In this example, $\tau=8$ and $\eta=3$.

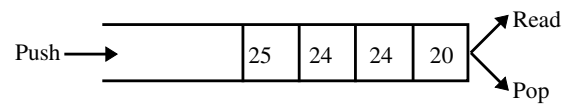

Fig. 3. A FIFO Queue containing time stamps. Timestamps are pushed behind the queue while they are read and popped from front of the queue.

The sequence $x_{t}$ is virtual in the sense that the operator may not have $x_{t}$ active leases at epoch $t$. Rather the operator decides $x_{t}$, for a past epoch $t$, by looking back in time and analyzing the number of additional channels it should have leased at epoch $t$ to minimize the loss. Similarly $y_{t}$ is the number of channels available for leasing at epoch $t$ if the operator had leased additional channels at past epochs. These two sequences helps the operator decide the number of channel it wants to lease in the current epoch. This can be explained as follows.

At current epoch $t$, the operator looks back $\tau$ epochs and find the first epoch after $t-\tau+1$ when an additional lease could have been bought (line 9.a.). Let this epoch be $t_{M}$. The net incremental renting cost $\mathcal{R}$ is the net renting cost which could have been saved in the time period $\left[t_{M}, t\right]$ if one more lease was bought in epoch $t_{M}$ (line 9.b.). Mathematically,

$$
\mathcal{R}=\sum_{i=t_{M}}^{t}\left[F_{i}\left(\left(D_{i}-x_{i}\right)^{+}\right)-F_{i}\left(\left(D_{i}-x_{i}-1\right)^{+}\right)\right]
$$

where $D_{i}$ and $x_{i}$ are the effective demand and the number of virtual active leases respectively in the $i^{\text {th }}$ epoch. $F_{i}\left(\left(D_{i}-x_{i}\right)^{+}\right)$and $F_{i}\left(\left(D_{i}-x_{i}-1\right)^{+}\right)$are the renting cost in the $i^{t h}$ epoch to serve the demands above the red and the blue graph respectively in Figure 2.

If $\mathcal{R} \geq P$ then the operator could have minimized the loss by leasing a channel in epoch $t_{M}$. To compensate for this mistake the operator decides to lease a channel. The current timestamp $t$ is pushed in the end of the FIFO queue as shown in Figure 3 indicating the decision to buy an additional lease (line 9.c.1.). A virtual lease is bought at epoch $t_{M}$ to indicate that a corrective measure has been taken for the past mistake. This updates the history of $x_{i}$ (line 9.c.2.) and $y_{i}$ (line 9.c.3.). Without such updation the operator will take corrective measure for the same mistake multiple times. The

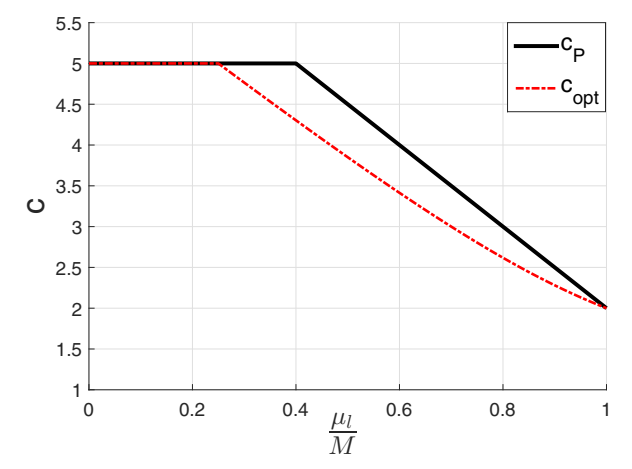

Fig. 4. A typical plot comparing the $\mathrm{CR}$ of $\mathcal{A}_{P}$ with the most optimal $\mathrm{CR}, c_{o p t}\left(\mu_{l}\right)$, as given by equation (12). In this plot $\frac{\tau p_{M}}{P}=5$.

future $x_{i}$ 's are also updated assuming that the operator can buy the additional lease in the current epoch (line 9.c.4.).

Step 3 is repeated till $\mathcal{R}<P$. When $\mathcal{R}<P$, it indicates that purchasing any more additional lease is costlier than renting. This is a direct consequence of Property 2. Hence the operator decides not to buy any additional leases.

\section{Step 4 (To lease or not)}

The timestamps of leasing decisions are read from the front of the FIFO queue (line 11.a.) as shown in Figure 3. If the wait time $\eta$ corresponding to the timestamp is lesser than $\tau-\frac{2 P}{p_{M}}$ (line 11.b), an additional channel is leased (line 11.b.1.). Finally the processed timestamp is popped out of the queue (line 11.c.). This step is continued either till the FIFO queue is empty or there are no more channels available for leasing in the current epoch.

A FIFO queue is used so that the timestamps are processed in the order in which they were generated. Otherwise it may happen that the wait time of a timestamp, which could have been below the threshold $\tau-\frac{2 P}{p_{M}}$ gets rejected because it was processed later.

\section{Step 5 (Calculate $o_{t}$ and $g_{t}$ )}

If there are $A_{t}$ active leases, then by Property 1 , $\left(D_{t}-A_{t}\right)^{+}$demands are served by renting (line 12). The number of channels to use opportunistically and the amount of demands to reject is given by equation (6) (line 13).

Theorem 3: The competitive ratio of online algorithm $\mathcal{A}_{P}$ $\left(\mathcal{A}_{z_{t h}}\right.$ with $\left.z_{t h}=P\right)$ is

$$
c\left(\mu_{l}\right)= \begin{cases}2+\frac{\tau p_{M}}{P}\left(1-\frac{\mu_{l}}{M}\right) & ; \mu_{l} \geq \frac{M}{\left(\frac{\tau p_{M}}{2 P}\right)} \\ \frac{\tau p_{M}}{P} & ; \mu_{l}<\frac{M}{\left(\frac{\tau p_{M}}{2 P}\right)}\end{cases}
$$

Proof: Please refer [14] for the proof.

Figure 4 compares the $\mathrm{CR}$ of $\mathcal{A}_{P}$ with $c_{\text {opt }}\left(\mu_{l}\right)$, the best possible CR that any online algorithm for OP3 (and hence OP1) can achieve. Figure 4 shows that the performance of $\mathcal{A}_{P}$ is satisfactorily close to the optimal CR.

\section{Simulation Results}

In this section we carry out simulations using artifically generated traces with two-fold objective. First, is to study the effect of few trace parameters on the online algorithm $\mathcal{A}_{P}$. Second, we compare $\mathcal{A}_{P}$ with some benchmark algorithms. 
TABLE II

COMMON TRACE Properties.

$1 \mathrm{EPOCH}=1 \mathrm{HOUR}, \tau=1 \mathrm{YEAR}, T=10 \tau, p_{M}=1, \frac{\tau p_{M}}{P}=5$, $M=50, d_{M}=15$. ' $*$ ' IMPLIES THAT THE FIELD CAN BE SET TO ANY ACCEPTABLE VALUE.

\begin{tabular}{|c|c|c|c|c|}
\hline Trace & \# of States & State Space & Mean $(\mu)$ & CV $\left(\frac{\sigma}{\mu}\right)$ \\
\hline$d_{t}$ & $d_{M}+1$ & $\left\{0, \ldots, d_{M}\right\}$ & 4 & 0.9 \\
\hline$M_{t}^{o}$ & $d_{M}+1$ & $\left\{0, \ldots, d_{M}\right\}$ & 2 & 0.5 \\
\hline$p_{t}$ & 50 & $\left\{0.8 p_{M}, \ldots, p_{M}\right\}$ & $0.95 p_{M}$ & 0.05 \\
\hline$\beta_{t}$ & 50 & $\{0.05, \ldots, 1\}$ & 0.66 & 0.35 \\
\hline$\lambda_{t}$ & $M+1$ & $\{0, \ldots, M\}$ & $\frac{3 M}{\tau}$ & $*$ \\
\hline$v_{t}$ & 3 & $\{0, \ldots, 2\}$ & $\frac{M}{1.5 \tau}$ & $*$ \\
\hline
\end{tabular}
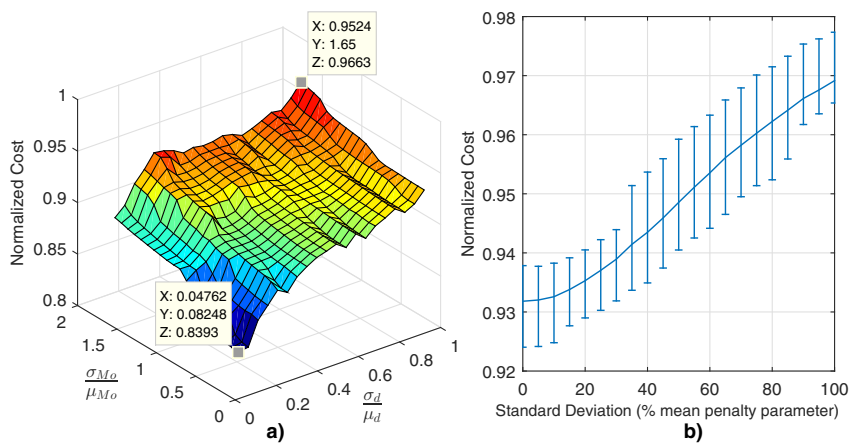

Fig. 5. a) The effect of the erratic nature of $d_{t}$ and $M_{t}^{o}$ on the normalized cost. The normalized cost for each pair of $\left(\frac{\sigma_{d}}{\mu_{d}}, \frac{\sigma_{M o}}{\mu_{M o}}\right)$ is averaged over 100 traces. $\mu_{d}$ and $\mu_{M o}$ was held constant throughout the simulation. b) Effect of erroneous $\beta_{t}$ on the normalized cost. The normalized cost for each value of standard deviation is averaged over 100 traces.

Setup and trace generation: Recall that $d_{t}, \lambda_{t}, p_{t}, M_{t}^{o}$, $v_{t}$ and $f_{t}(\cdot)$ are the inputs to $\mathbf{O P} 1$. In all our simulations, $f_{t}(o)=\frac{p_{t}}{2 M_{t}^{o} \beta_{t}} o^{2}$ if $M_{t}^{o}>0$ and 0 otherwise. $\beta_{t} \in(0,1]$ is the penalty parameter for opportunistic channel use. A lower $\beta_{t}$ implies higher penalty. Due to the lack of realworld traces, we had to generate artificial traces for $d_{t}, \lambda_{t}, p_{t}$, $M_{t}^{o}, v_{t}$ and $\beta_{t}$. We consider all the six traces to be discrete time markov chain (DTMC). The mean and coefficient of variation $\left(\mathrm{CV}^{8}\right)$ of the stationary distribution of all the six DTMC's (and hence the traces) can be controlled ${ }^{9}$. Table II tabulates common trace properties. These properties will be used in the following simulations unless stated otherwise.

Effect of erratic nature of $d_{t}$ and $M_{t}^{o}$ : The erratic nature of customer demand $d_{t}$ and opportunistic channel availability $M_{t}^{o}$ decides the value of available opportunistic channels. In this regard we study the following normalized cost: Cost incurred by $\mathcal{A}_{P}$ when it uses the opportunistic channels to the cost incurred by $\mathcal{A}_{P}$ when it does not use the opportunistic channels. An available opportunistic channel is of value only if there is a demand in that epoch, the probability of which decreases as $d_{t}$ and $M_{t}^{o}$ becomes erratic. Mathematically, the normalized cost should be monotonic increasing in $\frac{\sigma_{d}}{\mu_{d}}$ and $\frac{\sigma_{M o}}{\mu_{M o}}$ where $\mu_{d}$ and $\sigma_{d}\left(\mu_{M o}\right.$ and $\sigma_{M o}$ ) represents the mean and standard deviation of $d_{t}\left(M_{t}^{o}\right)$. As shown in

\footnotetext{
${ }^{8} \mathrm{CV}$ is the ratio of standard deviation to mean. It can be used as a measure of erratic nature of a trace. Higher the $\mathrm{CV}$, more erratic is the trace.

${ }^{9}$ The problem of designing a Markov matrix whose stationary distribution has a given mean and $\mathrm{CV}$ can be formulated as a linear program.
}
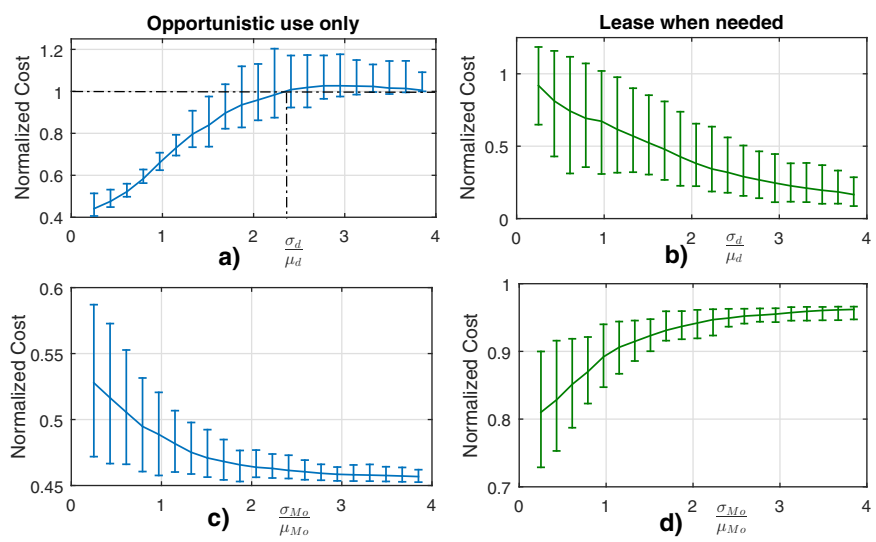

Fig. 6. The performance of our online algorithm with respect to two trivial algorithms. For each value of $\frac{\sigma}{\mu}$, we considered 4 values of $\mu$ and for each $(\sigma, \mu)$ pair, we averaged the normalized cost over 100 traces.

Figure 5.a., this intuition is verified by simulations too, subjected to simulation errors. There is a saving of $13 \%$ between the lowest and the highest $\left(\frac{\sigma_{d}}{\mu_{d}}, \frac{\sigma_{M o}}{\mu_{M o}}\right)$ pairs.

Effect of erroneous $\beta_{t}$ : Implementation of $\mathcal{A}_{P}$ relies on computing $f_{t}(o)$ which in turn relies on the knowledge of $\beta_{t}$. Penalty parameter $\beta_{t}$ depends on channel states like number of users in a given channel, the transmission power of individual users etc. The operator does not have direct access to these information, it can only infer it (possibly through customer feedback). Hence $\beta_{t}$ is prone to error. Understanding the effect of erroneous $\beta_{t}$ on the normalized cost (same as defined before) is important. To do this we add zero mean white gaussian noise to $\beta_{t}$ and compute the normalized cost incurred by $\mathcal{A}_{P}$ as we increase the standard deviation of the gaussian noise. This is shown in Figure 5.b. As expected, the normalized cost increases with increase in standard deviation. More importantly, with standard deviation as high as $100 \%$, the incurred cost can be reduced by $3 \%$ if we use the available opportunistic channels.

In the remaining part of this section, we will compare $\mathcal{A}_{P}$ with some benchmark algorithms. To do this we will use the following definition of normalized cost: Cost incurred by $\mathcal{A}_{P}$ to the cost incurred by the benchmark algorithm.

Comparison with trivial online algorithms: We compare $\mathcal{A}_{P}$ with two trivial online algorithms: i) Opportunistic use only: This algorithm never leases any channel. It uses available opportunistic channels and reject the remaining demand. ii) Lease when needed: This algorithm leases channels whenever the number of active channel leases is less then the demand, provided there are channels available for leasing. Leasing is not advisable if the demand is erratic because there is a high probability that the demand may decrease after we lease a channel. Therefore "opportunistic use only" works better when the demand is erratic (Figure 6.a.) and "lease when needed" works better when demand is smooth (Figure 6.b.). If the number of available opportunistic channel is erratic, it is better to lease a channel because there may not be opportunistic channels available in future. This intuition is validated by Figure 6.c. and 6.d. Figure 6 shows that $\mathcal{A}_{P}$ outperforms these trivial algorithms except when $\frac{\sigma_{d}}{\mu_{d}} \geq 2.3$. 


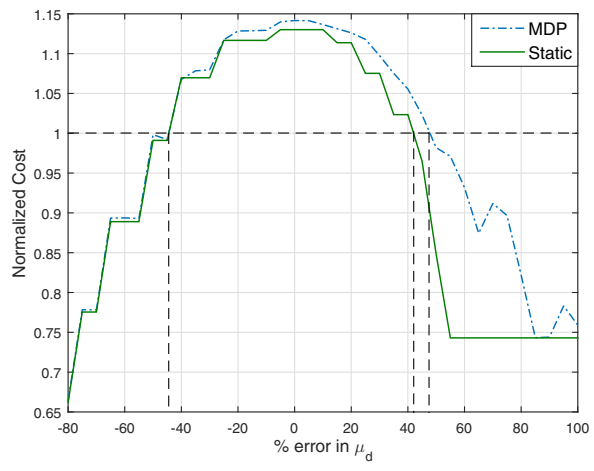

Fig. 7. The performance of our online algorithm with respect to two statistics based algorithms. For each \% error in $\mu_{d}$, the normalized cost has been averaged over 100 traces.

Comparison with statistics based online algorithms: To implement $\mathcal{A}_{P}$ we do not require any knowledge of the statistics of the six traces. Therefore $\mathcal{A}_{P}$ will be desirable in the early stages of the deployment of 3-TSF because knowledge of market statistics will be limited to none ${ }^{10}$. We illustrate the advantage of $\mathcal{A}_{P}$ by comparing it with two statistics based algorithms: i) Markov Decision Process $(M D P)$ : This algorithm was proposed in [3] and is the stateof-the-art work with any resemblance to our problem. It needs complete knowledge of the Markov matrices of all the traces. It can be implemented online only if $T \leq \tau^{11}$. In our case $T>\tau$ and hence we use the following heuristic: we divide the optimization horizon $T$ into $\frac{T}{\tau}$ frames and apply the algorithm to each frame seperately. ii) Static Leasing Strategy: This algorithm uses the stationary distribution of the traces to compute the number of active leases required to minimize the expected cost. It then tries to maintain the optimal number of active leases subjected to lease availability. Performance of such algorithms are prone to error in the statistical model. Figure 7 shows the normalized cost when $\mu_{d}$ is erroneous. As shown in Figure 7, $\mathcal{A}_{P}$ performes better than both the algorithms if $\mu_{d}$ is off by around $\pm 50 \%$. It is to be noted that in this simulation, all statistical parameter but $\mu_{d}$ was known accurately. Also due to the high time complexity of MDP, we could only simulate for $\tau=1$ week.

\section{Concluding Remarks And Extensions}

For a wireless operator who works in Tier-2 and Tier3 of the 3-TSF, it is important to strategically decide the number of channels to lease (Tier-2), the number of channels to use opportunistically (Tier-3) and the number of customer demands to reject. Such decisions rely on customer demand and channel availability pattern which can be considered as random processes. In this paper, we used tools from skirental literature to design an algorithm which makes online decisions without any knowledge of the statistics of the

\footnotetext{
${ }^{10}$ Statistics based algorithms like [3] will outperform $\mathcal{A}_{P}$ if market statistics is sufficiently accurate. Accurate market statistics will be available after the 3-TSF is in operation for a sufficiently long time.

${ }^{11}$ The MDP based algorithm has a linear time complexity if $T \leq \tau$ and psuedo-polynomial for $T>\tau$. Psuedo-polynomial time complexity is too high to be implementable online.
}

involved random processes. We claim that our algorithm will be of importance in the early stages of the deployment of 3-TSF because the operator will have either limited or no knowledge of market statistics. Our algorithm has bounded competitive ratio which is nearly optimal when compared with the least possible competitive ratio. In the process of designing an online algorithm for leasing channels, we formulated and studied the modified ski-rental problem which is state-of-the-art in ski-rental literature.

We are interested in addressing the following three issues in later works. First, the online algorithm for leasing channels which we designed has sub-optimal competitive ratio. We are interested in desiging an online algorithm which is optimal in sense of competitive ratio. Second, we are interested in designing randomized online algorithms for leasing channels. Third, we would like to explore other assumptions, like the lower bound on the time average of the number of channels available for leasing, through which we can derive a better bound on the competitive ratio.

\section{REFERENCES}

[1] The White House, "Realizing the full potential of governmentheld spectrum to spur economic growth," President's Council of Advisors on Science and Technology (PCAST) Report, July 2012. [Online]. Available: http://www.whitehouse.gov/sites/default/ files/microsites/ostp/pcast_spectrum_report_final_july_20_2012.pdf

[2] Federal Communications Commission, "Amendment of the commission's rules with regard to commercial operations in the 3550-3650 MHz band," May 2016. [Online]. Available: https://apps.fcc.gov/edocs_public/attachmatch/FCC-16-55A1.pdf

[3] G. Kasbekar, S. Sarkar, K. Kar, P. Muthuswamy, and A. Gupta, "Dynamic contract trading in spectrum markets," IEEE Trans. Autom. Control, vol. 59, no. 10, pp. 2856-2862, 2014.

[4] C. W. Kim, J. Ryoo, and M. M. Buddhikot, "Design and implementation of an end-to-end architecture for $3.5 \mathrm{GHz}$ shared spectrum," in Proc. IEEE DySPAN, 2015, pp. 23-34.

[5] C. Liu and R. A. Berry, "Competition and investment in shared spectrum," in Proc. IEEE Asilomar, 2015, pp. 643-647.

[6] K. Jagannathan, I. Menache, E. Modiano, and G. Zussman, "Noncooperative spectrum access-the dedicated vs. free spectrum choice," IEEE J. Sel. Areas Commun, vol. 30, no. 11, pp. 2251-2261, 2012.

[7] H. Kim, J. Choi, and K. G. Shin, "Hierarchical market competition in a duopoly super wi-fi spectrum market," IEEE J. Sel. Areas Commun, vol. 31, no. 11, pp. 2580-2591, 2013.

[8] L. Gao, J. Huang, Y.-J. Chen, and B. Shou, "An integrated contract and auction design for secondary spectrum trading," IEEE J. Sel. Areas Commun, vol. 31, no. 3, pp. 581-592, 2013.

[9] S. Anand, A. Sridharan, and K. N. Sivarajan, "Performance analysis of channelized cellular systems with dynamic channel allocation," IEEE Trans. Vehicular Tech., vol. 52, no. 4, pp. 847-859, 2003.

[10] L. Shi, K. W. Sung, and J. Zander, "Secondary spectrum access in tv-bands with combined co-channel and adjacent channel interference constraints," in Proc. IEEE DySPAN, 2012, pp. 452-460.

[11] A. R. Karlin, C. Kenyon, and D. Randall, "Dynamic TCP acknowledgement and other stories about e/(e-1)," in Proc. ACM STOC, 2001, pp. 502-509.

[12] W. Wang, B. Liang, and B. Li, "Optimal online multi-instance acquisition in IaaS clouds," IEEE Trans. Parallel Distrib. Syst., vol. 26 , no. 12, pp. 3407-3419, 2015.

[13] T. Lu, M. Chen, and L. L. Andrew, "Simple and effective dynamic provisioning for power-proportional data centers," IEEE Trans. Parallel Distrib. Syst., vol. 24, no. 6, pp. 1161-1171, 2013.

[14] G. Saha, A. A. Abouzeid, and M. Matinmikko, "Competitive online algorithm for leasing wireless channels in 3-tier sharing framework." [Online]. Available: https://www.ecse.rpi.edu/homepages/abouzeid/ preprints/comp_leasing.pdf

[15] J. Kiefer, "Sequential minimax search for a maximum," Proceedings of the American mathematical society, vol. 4, no. 3, pp. 502-506, 1953. 